\title{
FSTL1 interacts with VIM and promotes colorectal cancer metastasis via activating the focal adhesion signalling pathway
}

Chuansha Gu ${ }^{1,2}$, Xiaoyan Wang ${ }^{1,2,3}$, Ting Long ${ }^{1,2}$, Xia Wang ${ }^{1,2}$, Yan Zhong ${ }^{1,2}$, Yidan Ma ${ }^{1,2}$, Zhiyan $\mathrm{Hu}^{1,2,3}$ and Zuguo $\mathrm{Li}^{1,2,4}$

\begin{abstract}
Follistatin-like protein 1 (FSTL1) has been reported to have both tumour-promoting and tumour-suppressive characters. However, the role of FSTL1 in colorectal cancer (CRC) remains unclear. Here we showed that FSTL1 expression was significantly up-regulated in CRC tissues compared with the paired normal tissues. In addition, the higher FSTL1 expression was associated with the infiltrating depth, lymph node metastasis and poor prognosis of CRC. Enhanced expression of FSTL1 distinctly increased cell migration and invasion in vitro, as well as promoting liver metastasis of CRC in vivo. Conversely, knockdown of FSTL1 expression significantly repressed invasion and metastasis of CRC. Mechanically, transcription factor Smad3 was involved in FSTL1 protein expression inducing by TGF $\beta 1$-Smad2/ 3 signalling. Furthermore, this effect of FSTL1 in promoting CRC progression was actualised via activating focal adhesions signalling pathway and regulating cytoskeleton rearrangement. We identified VIM, as an interactive protein of FSTL1, participated in FSTL1-mediated aggressive phenotype. We showed the role of FSTL1 in CRC and explored its transcription regulation and downstream signalling molecular mechanisms. In conclusion, our findings suggested that FSTL1 promoted CRC progression and metastasis, making it a novel target for diagnosis and prognostic evaluation of CRC.
\end{abstract}

\section{Introduction}

Colorectal cancer (CRC) is one of the most common tumours and the third leading cause of cancer-related death in the world ${ }^{1,2}$. Although surgical techniques and adjuvant therapy have improved, the overall survival of patients with CRC has not improved obviously in recent years ${ }^{3,4}$. The main cause of mortality in patients with colorectal tumours is metastasis ${ }^{5}$, which is also a result of the accumulation of multiple genetic and epigenetic

\footnotetext{
Correspondence: Zhiyan Hu (zhenyucai2011@163.com) or

Zuguo Li (Lizg@smu.edu.cn)

'Department of Pathology, School of Basic Medical Sciences, Southern Medical University, Guangzhou, China

${ }^{2}$ Guangdong Provincial Key Laboratory of Molecular Tumour Pathology, Guangzhou, China

Full list of author information is available at the end of the article.

These authors have contributed equally: Chuansha Gu, Xiaoyan Wang.

Edited by M. Agostini
}

changes. Thus, a greater understanding of how molecular and cellular mechanisms of tumour progression combine to drive invasion and metastases in CRC is required.

Follistatin-like protein 1 (FSTL1), also refers as TSC-36 or FRP, is originally isolated in mouse osteoblastic MC3T3E1 cells as a cDNA that is up-regulated upon transforming growth factor-beta 1 (TGF- $\beta 1$ ) stimulation ${ }^{6}$. It is a 308 amino acid secreted glycoprotein, which belongs to the osteonectin (SPARC) family of proteins containing both extracellular calcium-binding and follistatin-like domains. FSTL1 is a protein with multiple regulatory functions, including regulating organ tissue formation in embryos ${ }^{7}$, regenerating the heart's cardiomyocytes $^{8}$. In particular, FSTL1 plays an important role in the inflammatory response to various pathogens. It activates immune cells and promotes gene expression and

\section{(c) The Author(s) 2018}

(c) (i) Open Access This article is licensed under a Creative Commons Attribution 4.0 International License, which permits use, sharing, adaptation, distribution and reproduction c. in any medium or format, as long as you give appropriate credit to the original author(s) and the source, provide a link to the Creative Commons license, and indicate if changes were made. The images or other third party material in this article are included in the article's Creative Commons license, unless indicated otherwise in a credit line to the material. If material is not included in the article's Creative Commons license and your intended use is not permitted by statutory regulation or exceeds the permitted use, you will need to obtain permission directly from the copyright holder. To view a copy of this license, visit http://creativecommons.org/licenses/by/4.0/. 
Table 1 The Relationship between FSTL1 expression and CRC clinicopathological features

\begin{tabular}{|c|c|c|c|c|}
\hline Characteristics & Low & High & $x^{2}$ value & $P$ value \\
\hline Frequency & 34 & 96 & & \\
\hline \multicolumn{5}{|l|}{ Gender } \\
\hline Male & 20 & 59 & 0.073 & 0.787 \\
\hline Female & 14 & 37 & & \\
\hline \multicolumn{5}{|l|}{ Age } \\
\hline$\leq 58$ & 12 & 47 & 1.891 & 0.169 \\
\hline$>58$ & 22 & 49 & & \\
\hline \multicolumn{5}{|c|}{ Tumour size (diameter in cm) } \\
\hline$<5$ & 23 & 66 & 0.039 & 0.843 \\
\hline$\geq 5$ & 11 & 29 & & \\
\hline \multicolumn{5}{|l|}{ Differentiation } \\
\hline Well & 9 & 14 & 2.436 & 0.119 \\
\hline Moderate_Poor & 25 & 82 & & \\
\hline \multicolumn{5}{|c|}{ Depth of tumour invasion } \\
\hline Mucosa-muscularis & 14 & 18 & 6.805 & 0.009 \\
\hline Full-thickness & 20 & 78 & & \\
\hline \multicolumn{5}{|l|}{ Lymph node status } \\
\hline Positive & 7 & 40 & 4.833 & 0.028 \\
\hline Negative & 27 & 56 & & \\
\hline \multicolumn{5}{|l|}{ Dukes' stage } \\
\hline Dukes $A+B$ & 24 & 55 & 1.862 & 0.172 \\
\hline Dukes $C+D$ & 10 & 41 & & \\
\hline
\end{tabular}

releases of proinflammatory cytokines ${ }^{9}$, and promotes fibrosis after lung injury ${ }^{10}$.

To some extent, inflammation is a key inducement of tumour progression. In view of the special role of FSTL1 in inflammation, the effect of FSTL1 in tumour progression has attracted the interest of researchers. FSTL1 is upregulated in a highly metastatic prostate cancer cell line and in most tumour tissue of glioblastoma multiforme patients ${ }^{11,12}$. FSTL1 promotes oncogenesis and metastasis in oesophageal squamous cell carcinoma by coordinating NF-kappa B and bone morphogenetic proteins (BMP) pathway ${ }^{13}$. Furthermore, it is a critical determinant governing cancer bone metastasis accompanied by expanding a population of pluripotent mesenchymal stem-like $\mathrm{CD}_{4} 5^{-} \mathrm{ALCAM}^{+}$cells derived from bone marrow ${ }^{14}$. On the contrary, this secreted glycoprotein is found to be down-regulated by using the primary cultures of metastatic and nonmetastatic clear-cell renal-cell carcinoma specimens for global gene-expression analysis ${ }^{15}$. FSTL1 is regarded as a tumour suppressor for inducing apoptosis of cancer cells in ovarian and endometrial cancers ${ }^{16}$. These results suggest that FSTL1 may have different regulatory effects in various tumour types. Therefore, the exact function of FSTL1 in the tumour needs to be further investigated.

So far, the role of FSTL1 in colorectal cancer progression and metastasis has not been well characterised. In the current study, we demonstrated the expression and clinical significance of FSTL1 in CRC samples, and then investigated the effects of aberrant expression on cellular biological behaviour by manipulating FSTL1 expression in CRC cells in vitro and in vivo. Importantly, we further explored the upstream and downstream regulation mechanism of FSTL1 in CRC progression and metastasis.

\section{Materials and methods \\ Clinical samples and cell lines}

Formalin-fixed paraffin embedded human colorectal carcinoma tissues $(n=130)$ for this study were obtained from the Department of Pathology, Nanfang Hospital, Southern Medical University, China. The clinical and pathological parameters of the patients were summarised in Table 1 . The fresh surgically resected CRC tissues and matched adjacent normal tissues $(n=12)$ were immediately frozen in liquid nitrogen until the future study. In each case, a diagnosis of primary CRC had been made, and none of them had received any preoperative chemotherapy or radiotherapy. Blood samples $(n=15)$ of CRC patients were obtained from Nanfang Hospital, and normal samples of healthy donors $(n=13)$ were obtained from the Southern Medical University, China. All specimens were collected with the informed consent of patients. The study was approved by the ethics committee of Nanfang Hospital, Southern Medical University, China.

Colorectal cancer cell lines, including Lovo, HT29, RKO, LS174T, Caco-2, DLD1, SW480, SW620, and a normal human foetal colonic mucosa cell line (FHC) were obtained from the Global Bioresource Centre (ATCC, USA). All cells were cultured in RPMI 1640 medium (Gibco, USA) supplemented with $10 \%$ foetal bovine serum (FBS) (Gibco, USA) at $37^{\circ} \mathrm{C}$ in a humidified atmosphere with $5 \% \mathrm{CO}_{2}$.

\section{Construction of stable cell lines}

Lentivirus vector carrying the luciferase gene (Luc) and the human FSTL1 sequence (FSTL1) or the FSTL1repressing shRNA sequence (GCCCAGTTGTTTGCTATCACT, FSTL1-sh) were purchased from Genechem (Shanghai, China). An empty vector (Vector) was used as control to FSTL1 overexpression. Lentivirus containing a scramble sequence (FSTL1-scramble) was used as control to FSTL1-shRNA. According to the manufacturer's instructions, stable cell lines were established by transfection of CRC cells with these lentivirus vectors. 
Antibiotic-resistant transfected cells were selected via puromycin (Sigma, USA) administration in the culture medium. FSTL1 transfection efficiency was assessed by quantitative real-time PCR (qRT-PCR) and western blotting, respectively.

\section{RNA extraction and qRT-PCR}

RNA extraction and qRT-PCR were performed, as previously described ${ }^{17}$. The primers for $\mathrm{qRT}-\mathrm{PCR}$ ae described in Supplementary Table S1.

\section{Western blotting}

Proteins extraction and western blotting were performed, as previously described ${ }^{17}$. The primary antibodies are displayed in Supplementary Table S2.

\section{Immunohistochemistry (IHC)}

IHC was performed on paraffin sections of CRC tissues according to standard LSAB protocol (Dako), using primary antibodies against FSTL1, TGF- $\beta 1$ (Proteintech, USA) respectively. The degree of staining in the sections was observed and scored independently by 2 pathologists. The percentage positivity of FSTL1 was scored from 0 to 3 , with 0 for $<10 \%, 1$ for $10-30 \%, 2$ for $31-50 \%$, and 3 for $>50 \%$. The staining intensity was scored as 4-point scale: 0 (no staining), 1 (weak staining, light yellow), 2 (moderate staining, yellowish brown), and 3 (strong staining, brown). Subsequently, FSTL1 expression was calculated as the multiplication value of percentage positivity score and staining intensity score, which ranged from 0 to 9 . The final expression level of FSTL1 was defined as low (0-4) and high (5-9) ${ }^{18}$.

\section{Enzyme linked immunosorbent assay (ELISA)}

FSTL1 levels in the serum of CRC patients and cell supernatant were measured by ELISA using a commercially available kit (Huamei Biotech, China), as described by the manufacturer. The results were expressed in $\mathrm{ng} / \mathrm{ml}$, and the optical density of the samples was compared with the standard curves.

\section{Coimmunoprecipitation (CoIP)}

Protein extracts from SW480 cells $(500 \mu \mathrm{g} / \mathrm{sample})$ were used to carry out the CoIP, as previously described ${ }^{17}$. The specific antibodies were displayed in Supplementary Table S2. Silver stain (Beyotime, China) was performed after immunoprecipitation WB and the different band was analysed by Quadrupole Mass Spectrometer (Thermo scientific, USA).

\section{Immunofluorescence and colocalization}

Immunofluorescent staining was performed, as previously described $^{17}$. The primary antibodies were displayed in Supplementary Table S2. 4, 6-Diamidino-2-phenylindole
(DAPI) was used to stain the cell nucleus, and phalloidin (Cytoskeleton, USA) was used to stain the F-actin. The immunofluorescence and the colocalization of FSTL1 or FAK and VIM were recorded using the laser scanning confocal biological microscope. The images were analysed by using FV10-ASW 3.0 Viewer.

\section{Dual-Luciferase activity assay}

Plasmid pGL3-WT containing wild-type FSTL1 promoter oligonucleotides (5'-AAGTCTGACTCCT-3') and pGL3-MUT with mutant target site (5'-AGACTCAACTCCT-3') was synthesised by Ruibiotech (Beijing, China). The sequences were predicted and designed according to bioinformatics analysis (http://jaspar. genereg.net/). $293 \mathrm{~T}$ cells and SW480 cells were seeded in 24-well plates $\left(1 \times 10^{5} /\right.$ well $)$ and cultured $24 \mathrm{~h}$ before transfection. PGL3-basic, pGL3-WT, and pGL3-MUT plasmids were co-transfected with pRL-TK (Promega, USA) and pCMV3-smad3 respectively using Lipofectamine 3000 (Invitrogen, USA). pRL-TK (Promega) vectors were used as control. After $48 \mathrm{~h}$, Luciferase activity was measured by the Dual-Luciferase Reporter Assay System (Promega, USA), as described by the manufacturer.

\section{Chromatin immunoprecipitation (ChIP)}

ChIP assays were performed according to ChIP-IT Kit (Active Motif, USA). Antibody against smad3 or IgG (Cell Signaling Technology, USA) was used to precipitate the DNA-protein complex and subsequently elute the DNA from the antibody. Primers specific for the FSTL1 promoter were 5'-CTGCCATTCCAGCCTTTA-3' (forward) and $5^{\prime}$-TGAGTGCCCACTGTTGTG-3' (reverse).The immunoprecipitated DNA was examined by PCR.

\section{Cell migration and wound-healing assay, invasion and 3D culture assay, and cell proliferation assay}

The migration and wound-healing assay, invasion and 3D culture assay, and cell proliferation of transfected CRC cells were determined as previously described ${ }^{19}$.

\section{Tumour metastasis assay in vivo}

4-5 week-old $\mathrm{Balb} / \mathrm{C}$-nu/nu athymic mice $(n=5$ per group) were obtained from Animal Centre of Guangdong Province (permit number: SYXK2016-0167). All animal experiments were approved by the Institutional Animal Care and Use Committee of Southern Medical University. Considering that FSTL1 may be affected by oestrogen, we chose male nude mice as the research objects. To investigate the tumour metastasis in vivo, $5 \times 10^{6}$ of RKO cells stably expressed human FSTL1 sequence or empty vector carrying luciferase label in a volume of $75 \mu \mathrm{l}$ in PBS were injected into spleen subcapsular, respectively. Multimodal Image Station System (Bruker, Germany) was used to obtain images of tumour progression and liver metastasis. 
The images were taken at 10 minutes after intraperitoneal injection of $150 \mu \mathrm{g} / \mathrm{Kg}$ D-Luciferin potassium salts (Synchem, USA). All the images were analysed by Bruker MI SE 721 Installer. After 8 weeks, the spleens and livers of nude mice were surgically removed after euthanasia, fixed in formalin (neutral buffered 10\%), embedded in paraffin, and prepared into $3-\mu \mathrm{m}$ sections for hematoxylin-eosin (HE) staining and IHC analysis.

\section{Statistical analysis}

All statistical analyses were performed using the SPSS 19.0 (Abbott Laboratories, USA). All results were confirmed by statisticians in the Department of Health Statistics, Southern Medical University. The significance of correlation between the expression of FSTL1 and histopathological factors was determined using Pearson $\chi^{2}$ test. Survival analysis was carried out using the Kaplan-Meier method, and the log-rank test was used to compare the survival curves. Comparisons between groups were performed with a 2-tailed paired Student's $t$-test. In vitro cell growth assay was tested using factorial design ANOVA. ${ }^{*} P<0.05, \quad * * P<0.01$ and ${ }^{* * *} P<0.001$ were considered statistically significant.

\section{Results}

FSTL1 expression in CRC correlates with tumour invasiveness and poor prognosis

Western blotting and qRT-PCR were utilised to test the expression of FSTL1 in CRC cell lines and the normal colonic mucosa cell line (FHC). Our results revealed that FSTL1 was up-regulated in all the 8 CRC cell lines at the protein and mRNA level. (Fig. 1a, b). Immunohistochemistry (IHC) staining was performed in 130 paraffin-embedded CRC tissues sections. As shown in Fig. 1c, different levels of FSTL1 expression were observed in these tissue samples. According to classification as detailed described in method, overexpression of FSTL1 was detected in 96 out of 130 (73.8\%) CRC cases compared with 34 out of 103 (33.0\%) in adjacent non-tumour tissues. FSTL1 expression score was also higher in tumour tissues $(P<0.0001$, Fig. $1 \mathrm{~d})$. The correlation between FSTL1 expression level and CRC clinical features was further analysed (Table 1). The data indicated that overexpression of FSTL1 was closely related to depth of tumour invasion $(P=0.009)$, and lymph node metastasis $(P=0.028)$. Moreover, FSTL1 expression in 12 CRC tissues $(\mathrm{T})$ and paired normal colorectal mucosa $(\mathrm{N})$ was detected by western blotting. As a secreted protein, FSTL1 level in the serum was measured by ELISA. Consistent with the tissues samples, higher FSTL1 expression of serum was found in 15 CRC patients compared with 13 healthy donors $(P=0.046$, Fig. 1e). As shown in Fig. 1f, an increase in FSTL1 expression was observed in CRC tissues compared with adjacent normal tissues $(P=0.0178)$.
In addition, to investigate whether the different levels of FSTL1 expression in CRC are related to patient's prognosis, we performed bioinformatic analysis of NCBI GEO Database (GSE17536, $n=177$ ). Kaplan-Meier survival analysis revealed that patients with a higher level of FSTL1 expression had a worse clinical outcome $(P=$ 0.003 , Fig. 1g). These observations demonstrate that FSTL1 is up-regulated in CRC and correlates with its depth of tumour invasion, lymph node metastasis and poor prognosis.

FSTL1 promotes CRC cells migration and invasion in vitro

To gain insight into the effect of FSTL1 on cellular behaviour in CRC tumourigenesis and progression, we generated DLD1-FSTL1 and RKO-FSTL1 cell lines that were stably overexpressed full-length FSTL1. Furthermore, Lovo-FSTL1-sh and SW480 FSTL1-sh cell lines that stably expressed FSTL1-shRNA were made. The expression level of FSTL1 in stable lines was verified by western blotting analysis and qRT-PCR (Figs. 2a and 3a, Supplementary Figure S1a and S2a).

Since FSTL1 expression was related to CRC invasion depth and lymph node metastasis as described, trans-well and wound-healing assays were performed. As shown in Fig. 2b, c, the migration and motility ability of DLD1FSTL1 cells $(P<0.0001, P=0.03)$ and RKO-FSTL1 cells $(P<0.0001, P=0.0052)$ were enhanced compared with the control cells. Matrigel-coated Boyden chamber invasion assay revealed that overexpression of FSTL1 significantly promoted the invasive ability of CRC cells in vitro (both $P<0.0001$, Fig. 2e). Conversely, knockdown of FSTL1 slowed the migration and motility ability of Lovo-FSTL1-sh cells $(P<0.0001, P=0.0001)$ and SW480 FSTL1-sh cells $(P<0.0001, P=0.0203)$, and decreased the invaded cell numbers (both $P<0.0001$, Fig. 3b, c, e). Moreover, to further validate the effect of FSTL1 on cell invasion, we performed three-dimensional culture assays. Different stable cells were seeded in threedimensional Matrigel cultures at a same density and their colony shapes were examined 10 days later. Expectedly, FSTL1 over-expressing cells were highly invasive and extended protuberances to the Matrigel matrix, and the control cells formed tight spherical colonies (Fig. 2d). On the contrary, knockdown of FSTL1 inhibited the invasive phenotype (Fig. 3d). Neither overexpression nor knockdown of FSTL1 showed any significant difference in the capacity of CRC cells proliferation by CCK- 8 assay (Supplementary Figure. S1b and S2b). These data indicate that FSTL1 promotes CRC cellular migration and invasion in vitro, but has no effect on CRC cells proliferation.

\section{FSTL1 overexpression enhances CRC metastasis in vivo}

The effect of FSTL1 on tumour metastasis was assessed by spleen subcapsular injection of human CRC cells into 


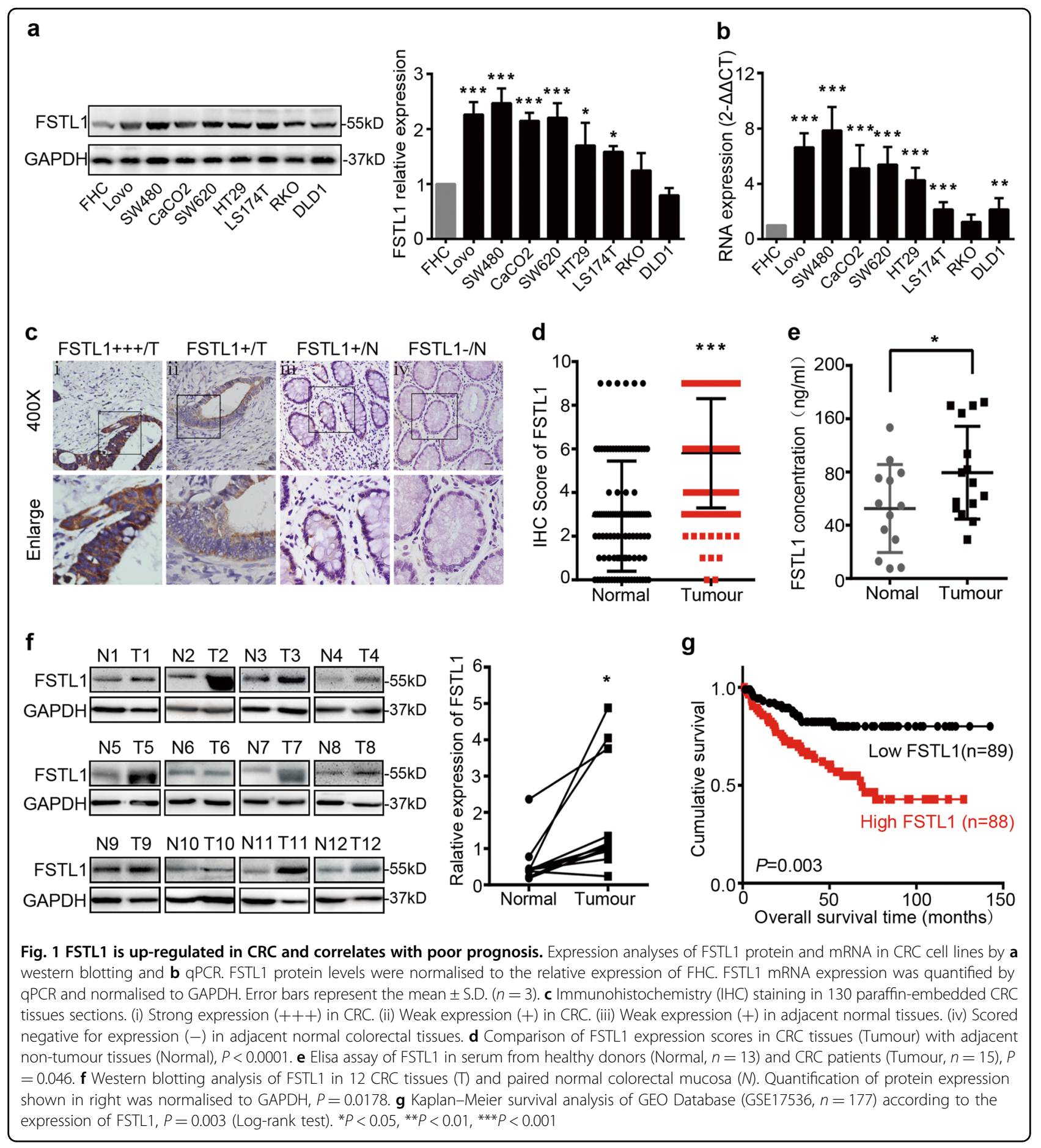

nude mice. Fig. 4a showed two typical pictures of each group on every 10 days after injection. In order to observe more easily, the left lateral position of the mouse was used at 10 and 20 days, and the prone position was used after 30 days. Xenograft tumours in spleen and metastatic nodules in liver were earlier detected in the group of FSTL1 overexpression (FSTL1) compared with those in the control group (Vector). 8 weeks after injection, larger tumours of spleens and more liver metastatic nodules were observed in the FSTL1 group in gross morphology (Supplementary Figure. S3). As shown in Fig. 4b, IHC staining confirmed that the tumours derived from FSTL1 group exhibited higher FSTL1 expression levels than tumours derived from control cells. Significant difference was revealed in photons $/ \mathrm{s} / \mathrm{cm}^{2}$ between the FSTL1 group and Vector group from 20 days after injection (Fig. 4c). 


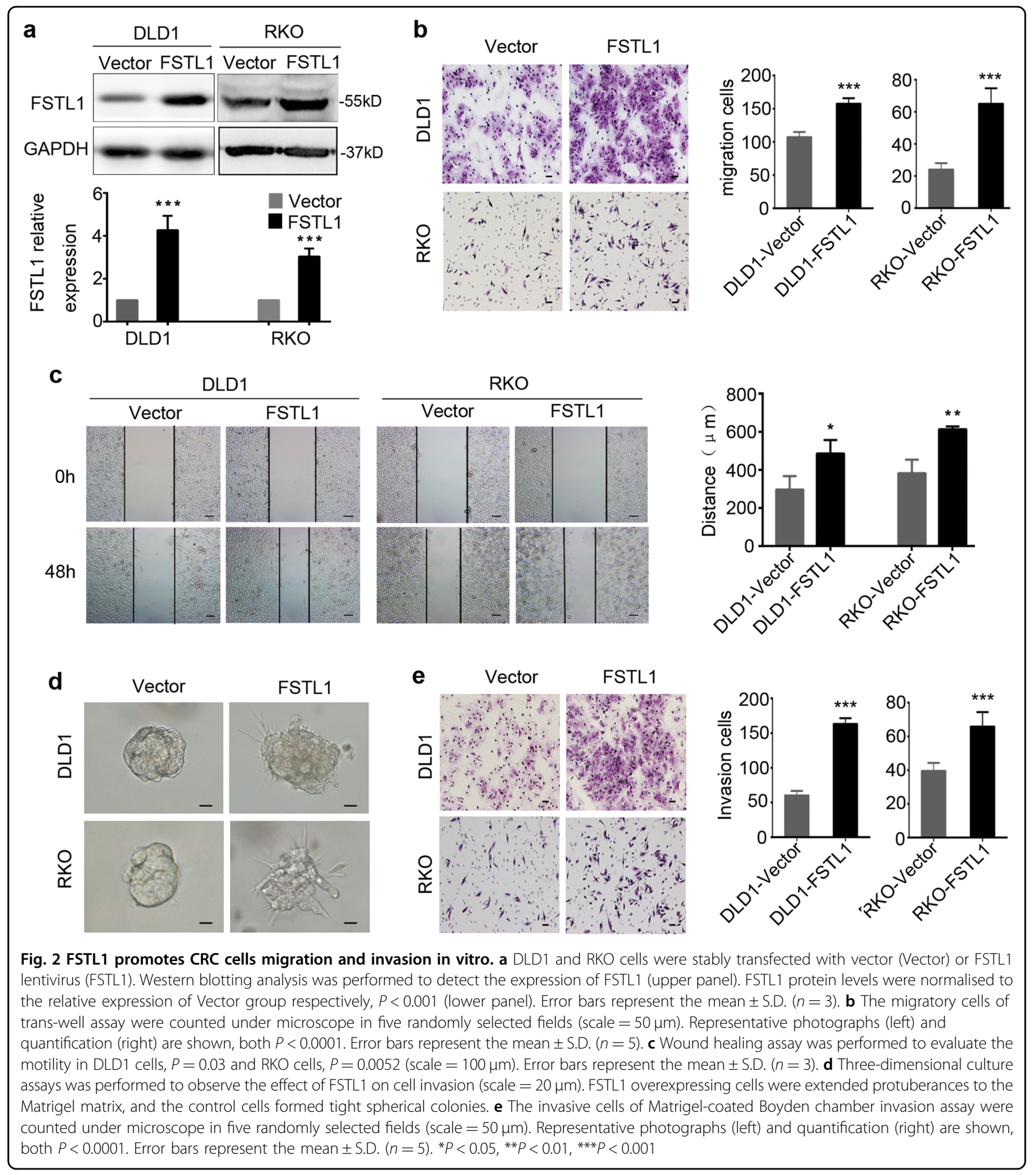

Moreover, the number of liver metastatic foci of the FSTL1 group was more than the Vector group $(P=0.010$, Fig. 4d). In addition, overexpression of FSTL1 shortened the survival time of nude mice $(P=0.041$, Fig. 4e). These results reveal that overexpression of FSTL1 significantly enhances metastasis of CRC cells in vivo.
TGF $\beta 1-S m a d 2 / 3$ signalling pathway regulates the expression of FSTL1 through activating the transcriptional activity of Smad3 in human CRC

Although FSTL1 was initially identified as TGF- $\beta 1$ induced protein in a murine cell line, the molecular mechanisms underlying its expression in human CRC is 


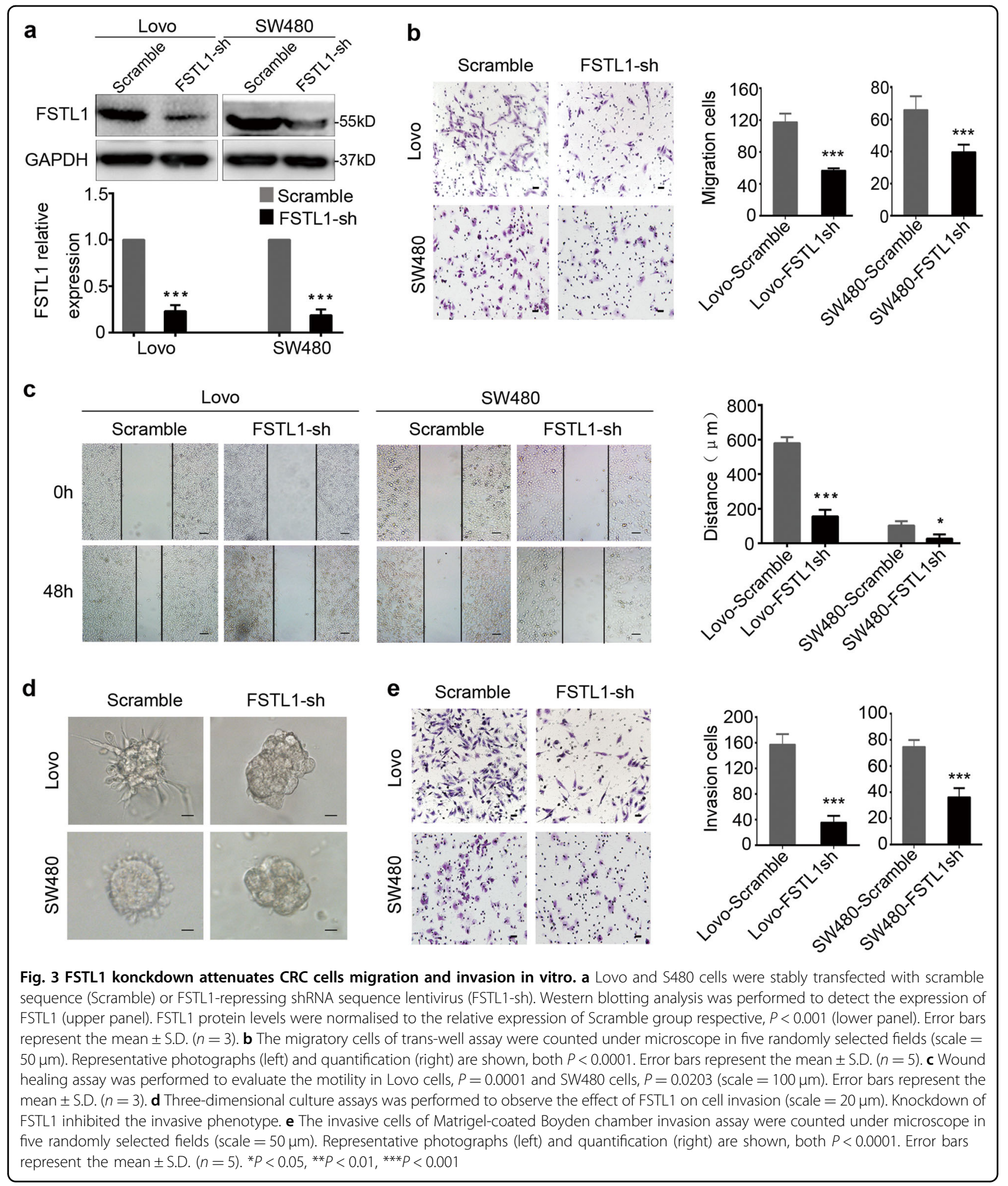

not yet understood. As shown in Fig. 5a, FSTL1 and TGF$\beta 1$ protein expression levels in consecutive paraffinembedded slice of human CRC tissue were detected by IHC. The bioinformatic analyses indicated that there was positive correlation between FSTL1 and TGF- $\beta 1$. (http:// r2.amc.nl, Supplementary Figure. S4a). CRC cell lines DLD1 and RKO with lower FSTL1 expression were treated with recombinant human TGF- $\beta 1$ (rhTGF $\beta-1)$. 


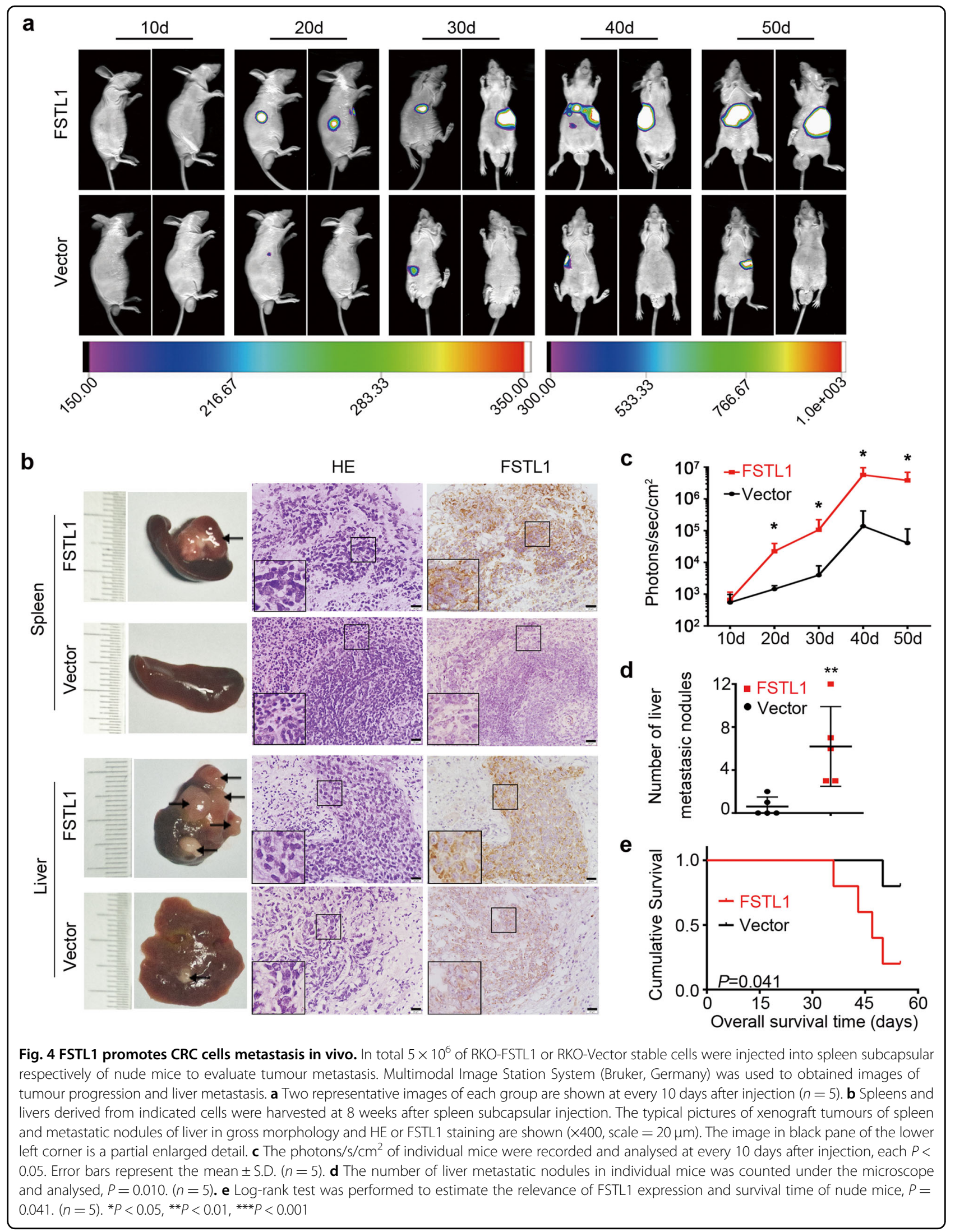




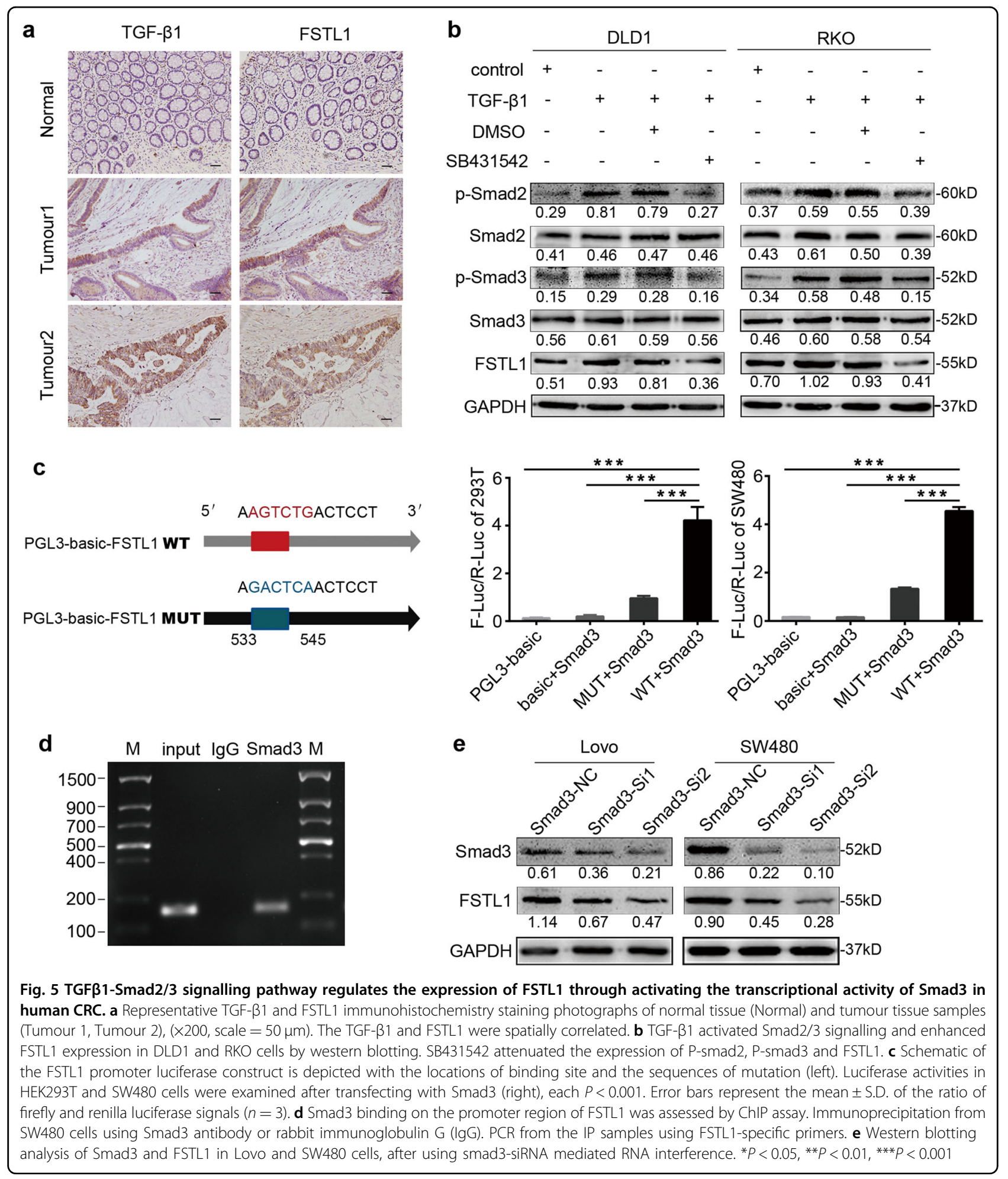

Western blotting analysis indicated that TGF- $\beta 1$ enhanced Smad2/3 phosphorylation (P-Smad2/3) and FSTL1 expression. While blocking TGF- $\beta$ signal transduction by SB431542, a specific inhibitor of Smad2/3, TGF- $\beta 1$-induced FSTL1 protein expression decreased (Fig. 5b). Meanwhile, neither overexpression nor knockdown of FSTL1 significantly changed the phosphorylation levels of smad2 and smad3 in stable CRC cells (Supplementary Figure S4b). 
To further explore the upstream mechanism of FSTL1 expression, potential transcription factors promoting were analysed by bioinformatic algorithms. It showed that Smads $(\operatorname{smad} 2 / \operatorname{smad} 3 / \operatorname{smad} 4)$ is the predicted transcription factor with binding site on the FSTL1 promoter at 533 to $545 \mathrm{bp}$ (including GTCT box) in the JARSPAR databases. Smad3 can be combined directly with DNA, whereas Smad2 can not, because Smad2 has 2 amino acid fragments more than $\mathrm{Smad}^{20}$. So we performed dualluciferase activity assay to verify whether smad3 participated in regulating the expression of FSTL1. As shown in Fig. 5c, compared with other groups, the ratio of firefly and renilla luciferase signals increased significantly in WT group which was transfected with plasmid pGL3-WT containing wild-type FSTL1 promoter oligonucleotides $(P<0.0001)$. In addition, ChIP assays demonstrated that smad3 bound to FSTL1 promoter (Fig. 5d). Subsequently, Western blotting was carried out in Lovo and SW480 cells using Smad3-siRNA mediated RNA interference. The expression of FSTL1 reduced after knockdown of Smad3 (Fig. 5e). Collectively, these data reveal that TGF 31 Smad2/3 signalling pathway regulates FSTL1 expression through activating the transcriptional activity of transcription factor Smad3.

\section{FSTL1 activates the focal adhesion signalling pathway and} regulates cytoskeleton rearrangement

To understanding the molecular mechanism of FSTL1 in CRC progression, the CRC data were downloaded from GEO public database and Gene Set Enrichment Analysis (GSEA) was carried out. KEGG-Focal adhesion, KEGGRegulation of actin cytoskeleton and PID-Integrin1 pathway gene sets were positive enriched (Fig. 6a). Western blotting analysis was performed to detect the levels of FSTL1 and the main members of focal adhesion signalling pathway, including phospho-FAK (Tyr-397), FAK, phospho-Paxillin (Tyr-118), Paxillin, phospho-SRC (Tyr418), SRC and Integrin- $\beta 1$. As shown in Fig. 6b, accompanied by changes of FSTL1 protein expression, these major molecules have occurred corresponding changes. Next, we explored whether FSTL1 had an effect on regulation of actin cytoskeleton. As shown in Fig. 6c,d and Supplementary Figure. S5, immunofluorescent staining showed that the pattern of F-actin changed from spikelike squiggles or short nailed protuberances to long stress fibres in the FSTL1 overexpression cells compared with control cells. Furthermore, the expression of FAK enhanced in FSTL1 overexpression group compared with the control group. Interestingly, phospho-FAK concentrated from the cytoplasm to the edge and formed more focal adhesions in the FSTL1 overexpression cells. These results suggest that FSTL1 takes part in cell adhesion and regulate cytoskeleton rearrangement via activating focal adhesion signalling pathway.

\section{VIM is an interactive factor of FSTL1}

To identify unknown interactive molecules of FSTL1, the whole proteins of whole-cell lysate from SW480 cells was extracted and immunoprecipitated with FSTL1 antibody (Fig. 7a). As shown in Fig. 7b, the most clearly differential protein band was identified as VIM by liquid chromatography-mass spectrometry. According to the results, we conjectured that FSTL1, VIM and FAK might form a complex. Thus protein samples were confirmed by CoIP with antibodies against FSTL1 or VIM (Fig. 7c). The IF assays validated localisation of VIM and its partial colocalisation with FSTL1 (Fig. 7d, upper panel). The colocalisation coefficients of VIM and FSTL1 in RKO cells and DLD1 cells are $0.473 \pm 0.023(n=10)$ and $0.431 \pm$ $0.050(n=10)$, respectively. Previous studies have reported that VIM enters into focal adhesions sites and regulates cell adhesion ${ }^{21,22}$. The interaction between VIM and FAK was proved by CoIP and colocalisation (Fig. 7d, lower panel). The colocalisation coefficients of VIM and FAK in RKO cells and DLD1 cells are $0.576 \pm 0.045(n=$ $10)$ and $0.505 \pm 0.023(n=10)$, respectively.

\section{Discussion}

In the light of the high morbidity and mortality of colorectal cancer, efforts to elucidate new biomarkers and more effective therapies are still imperative in order to improve survival for CRC patients. FSTL1 has been reported in the fields of inflammation, postischemia vascular remodelling, immune regulation and autoimmune diseases in recent years ${ }^{9}$. Oncomine analysis (https:// www.oncomine.org) showed that the expression of FSTL1 varies according to the organs and systems. It is upregulated in some tumour types, including brain and CNS cancer, colorectal cancer, pancreatic cancer, prostate cancer and so on. So far, the explicit expression and particular function of FSTL1 in human CRC still remain obscure. In the present study, we investigated the expression of FSTL1 in a clinical cohort with 130 cases. Our data verified that the overexpression of FSTL1 in CRC tissues was associated with the depth of tumour infiltration and the distant metastasis. A significant correlation was found between FSTL1 overexpression and CRC patient poor survival. Furthermore, due to FSTL1 is a secreted protein, it is detectable in the blood serum of patients. Our data showed that FSTL1 in serum of CRC patients was higher than that in health donors. These observations support the notion that FSTL1 may serve as a valuable biomarker in tumorigenesis and progression of CRC.

As an inflammatory cytokines, FSTL1 could be induced in vitro in osteoblasts, adipocytes, chondrocytes, and human fibroblast-like synoviocytes by IL-1 $\beta$, TNF $\alpha$, and IL-6. The NF-KB pathway was involved in the induction of FSTL1 gene transcription ${ }^{23,24}$. Moreover, using an 


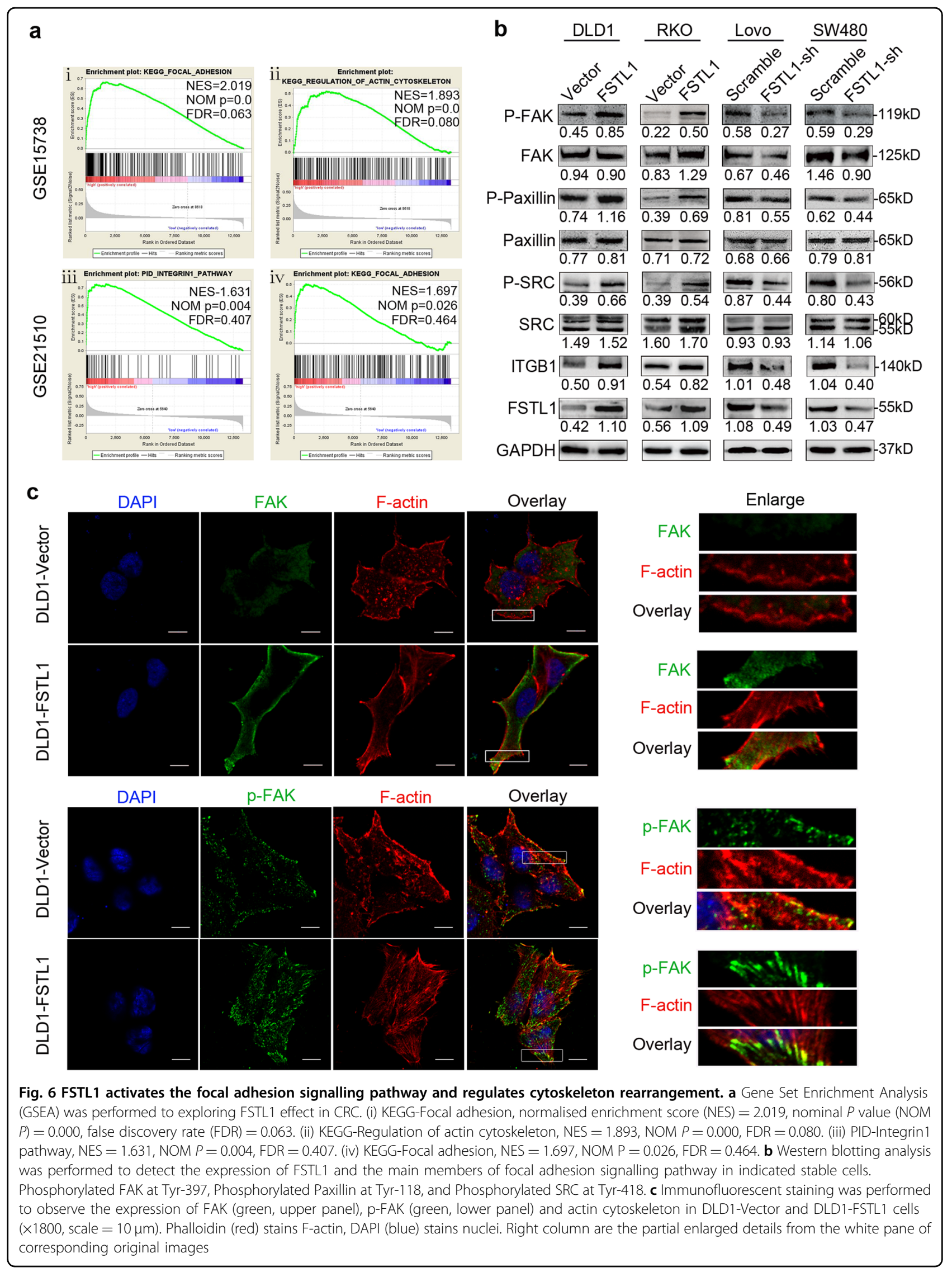




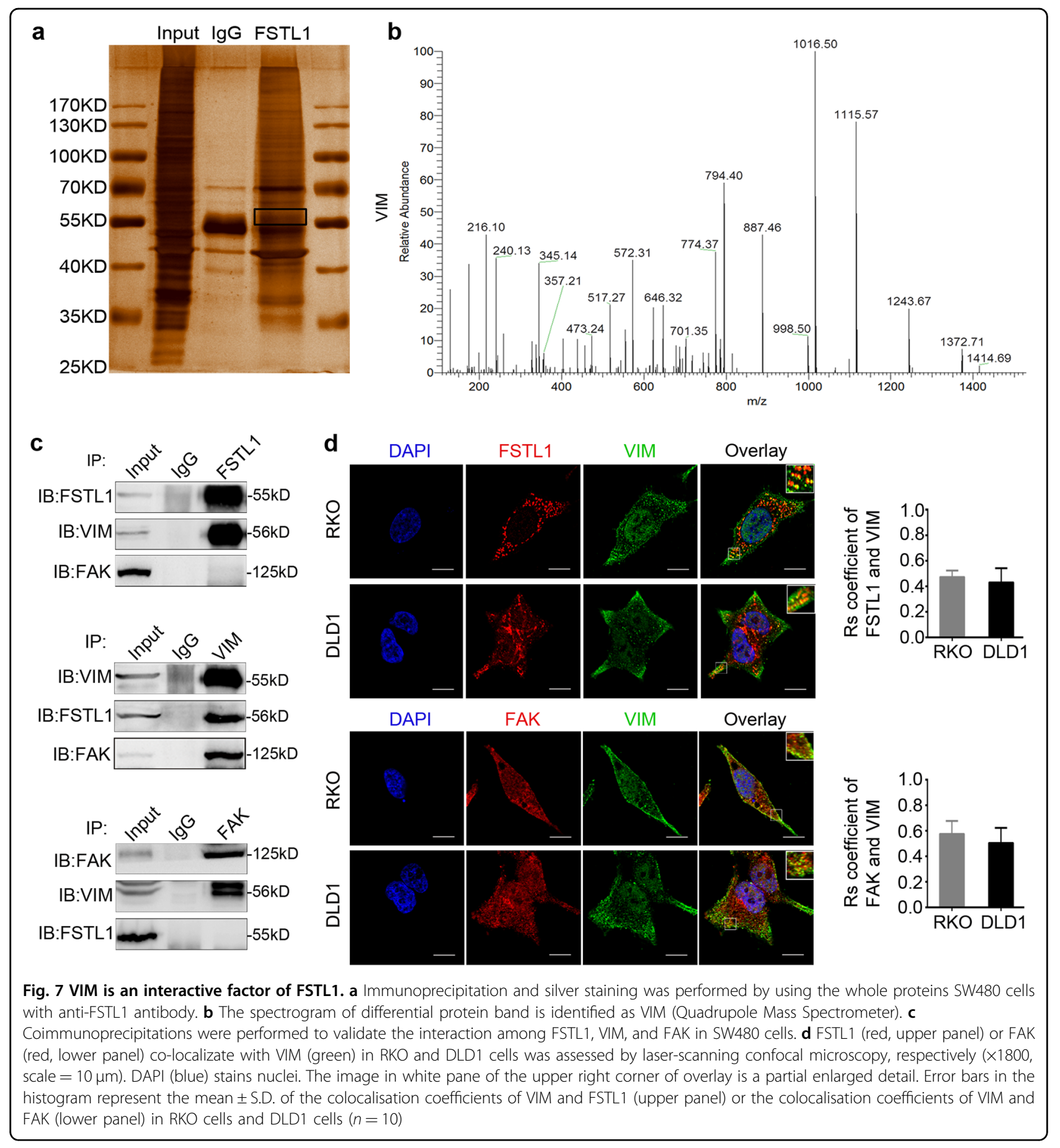

inducible Akt1 transgenic mouse model, researchers found that Fstl1 protein and transcript expression are increased by Akt activation in the heart ${ }^{25}$. However, the regulation of FSTL1 expression in human tumours had hitherto not fully elucidated. As already mentioned, FSTL1 was cloned as a TGF- $\beta 1$ inducible gene. Meanwhile, TGF- $\beta 1$ is well known to be regarded as a metastasis inducer, participating in malignant progression and angiogenesis ${ }^{26}$. Therefore, we verified the consistency of the expressions of FSTL1 and TGF- $\beta 1$ in CRC tissues. More importantly, we firstly demonstrated in some detail that TGF $31-S m a d 2 / 3$ signalling pathway regulated FSTL1 expression through activating the transcriptive activity of transcription factor Smad3, which combined with the 
promoter of FSTL1 directly in human CRC cells. These observations might present an opportunity to exploit such a mechanism for intervention of FSTL1 and therapeutic gain.

FSTL1 has proven to be able to enhance CRC cells migration and invasion in vitro and promote liver metastasis of CRC in vivo in the present study. We further investigated its downstream mechanism and interactive molecular targets. KEGG-Focal adhesion, KEGGRegulation of actin cytoskeleton and PID-Integrin1 pathway gene sets were positive enriched by GSEA. Focal adhesion kinase (FAK) is a cytoplasmic protein tyrosine kinase that is overexpressed and activated in several invasive tumours ${ }^{27}$. FAK is at the intersection of various signalling pathways promoting cancer cell motility $^{28}$, invasion ${ }^{29}$, cell survival ${ }^{30}$, and epithelialmesenchymal transition (EMT) ${ }^{31}$. The Tyr-397 phosphorylation and kinase activity of FAK are substantiated to be essential for the invasive phenotype ${ }^{32}$. Our results demonstrated that FSTL1 activated FAK focal adhesion signalling pathway in CRC cells, and followed by regulating cytoskeleton rearrangement. It suggested that focal adhesion signalling was involved in the FSTL1mediated acceleration of CRC progression.

We further explored the proteins interacting with FSTL1 via a protein-protein interaction technique. Among the candidate proteins, VIM was identified as a novel target of FSTL1. VIM, a major constituent of the intermediate filament family of proteins, is widely known as a marker for EMT. It correlates with accelerated tumour growth, invasion, and poor prognosis. In addition, it is considered to be a regulator of focal adhesions (FAs) and motility ${ }^{33}$. VIM assembles into focal adhesions and it is required for FAK activity and localisation at the cellular leading edge of motile cancer cells ${ }^{22,34,35}$. VIM assembly into focal adhesions is an important factor of cell adhesion strength and its overexpression enhances FAs turnover ${ }^{36}$. The results of CoIP showed that there was no interaction between FSTL1 and FAK, while VIM had interaction with FSTL1 and FAK, respectively. Therefore, we speculated that VIM might act as a media. The detailed mechanisms about how the interaction of FSTL1 and VIM is involved in focal adhesion signalling pathway in CRC cells will be interesting subjects for the future study.

In summary, we demonstrated that FSTL1 was upregulated and had a close relationship with poor outcome in CRC. The overexpression of FSTL1, inducing by TGF $\beta 1-S m a d 2 / 3$ signalling pathway, functionally promoted CRC cells migration, invasion, and metastasis by combining with VIM and activating focal adhesion signalling pathway consequently. Therefore, our data provide evidence for FSTL1 being regarded as a detectable biological marker that reflects malignant degree and evaluates survival in CRC.

\section{Acknowledgements}

This work was supported by grants from the National Natural Science Foundation of China (Nos. 81201664, 81672437 and 81702894). This research was also supported in part by the Provincial Science Foundation of Guangdong (2014A030313304, 2017A030310116).

\section{Author details}

'Department of Pathology, School of Basic Medical Sciences, Southern Medical University, Guangzhou, China. ${ }^{2}$ Guangdong Provincial Key Laboratory of Molecular Tumour Pathology, Guangzhou, China. ${ }^{3}$ Department of Pathology, Nanfang Hospital, Southern Medical University, Guangzhou, China.

${ }^{4}$ Department of Pathology, Shenzhen Hospital of Southern Medical University, Shenzhen, China

\section{Conflict of interest}

The authors declare that they have no conflict of interest.

\section{Publisher's note}

Springer Nature remains neutral with regard to jurisdictional claims in published maps and institutional affiliations.

Supplementary Information accompanies this paper at (https://doi.org/ 10.1038/s41419-018-0695-6).

Received: 3 January 2018 Revised: 30 April 2018 Accepted: 10 May 2018 Published online: 29 May 2018

\section{References}

1. Siegel, R., Desantis, C. \& Jemal, A. Colorectal cancer statistics, 2014. CA Cancer J. Clin. 64, 104-117 (2014)

2. Carethers, J. M. \& Jung, B. H. Genetics and genetic biomarkers in sporadic colorectal cancer. Gastroenterology 149, 1177-1190.e1173 (2015).

3. Edwards, B. K. et al. Annual report to the Nation on the status of cancer, 1975-2010, featuring prevalence of comorbidity and impact on survival among persons with lung, colorectal, breast, or prostate cancer. Cancer 120, 1290-1314 (2014).

4. Marz, L. \& Piso, P. Treatment of peritoneal metastases from colorectal cancer. Gastroenterol. Rep. 3, 298-302 (2015).

5. Brenner, H., Kloor, M. \& Pox, C. P. Colorectal cancer. Lancet 383, 1490-1502 (2014).

6. Shibanuma, M., Mashimo, J., Mita, A., Kuroki, T. \& Nose, K. Cloning from a mouse osteoblastic cell line of a set of transforming-growth-factor-beta 1regulated genes, one of which seems to encode a follistatin-related polypeptide. Eur. J. Biochem. 217, 13-19 (1993).

7. Adams, D., Larman, B. \& Oxburgh, L. Developmental expression of mouse Follistatin-like 1 (Fstl1): dynamic regulation during organogenesis of the kidney and lung. Gene. Expr. Patterns 7, 491-500 (2007).

8. Rochette, L. \& Vergely, C. "Pro-youthful" factors in the "labyrinth" of cardiac rejuvenation. Exp. Gerontol. 83, 1-5 (2016).

9. Chaly, Y., Hostager, B., Smith, S. \& Hirsch, R. Follistatin-like protein 1 and its role in inflammation and inflammatory diseases. Immunol. Res. 59, 266-272 (2014).

10. Dong, $Y$. et al. Blocking follistatin-like 1 attenuates bleomycin-induced pulmonary fibrosis in mice. J. Exp. Med. 212, 235-252 (2015).

11. Trojan, L. et al. Identification of metastasis-associated genes in prostate cancer by genetic profiling of human prostate cancer cell lines. Anticancer Res. 25 183-191 (2005).

12. Reddy, S. P. et al. Novel glioblastoma markers with diagnostic and prognostic value identified through transcriptome analysis. Clin. Cancer Res. 14, 2978-2987 (2008).

13. Lau, M. C. et al. FSTL1 promotes metastasis and chemoresistance in esophageal squamous cell carcinoma through NFkappaB-BMP signaling cross-talk. Cancer Res. 77, 5886-5899 (2017)

14. Kudo-Saito, C., Fuwa, T., Murakami, K. \& Kawakami, Y. Targeting FSTL1 prevents tumor bone metastasis and consequent immune dysfunction. Cancer Res. 73, 6185-6193 (2013).

15. Tan, X. et al. Global analysis of metastasis-associated gene expression in primary cultures from clinical specimens of clear-cell renal-cell carcinoma. Int. J. Cancer 123, 1080-1088 (2008). 
16. Chan, Q. K. et al. Tumor suppressor effect of follistatin-like 1 in ovarian and endometrial carcinogenesis: a differential expression and functional analysis. Carcinogenesis 30, 114-121 (2009).

17. Hu, Z. Y. et al. AKAP-9 promotes colorectal cancer development by regulating Cdc42 interacting protein 4. Biochim. Biophys. Acta 1862, 1172-1181 (2016).

18. Gu, Y. et al. TUSC3 promotes colorectal cancer progression and epithelialmesenchymal transition (EMT) through WNT/beta-catenin and MAPK signalling. J. Pathol. 239, 60-71 (2016).

19. Yang, M. H. et al. MALAT1 promotes colorectal cancer cell proliferation/ migration/invasion via PRKA kinase anchor protein 9. Biochim. Biophys. Acta 1852, 166-174 (2015)

20. Shi, Y. et al. Crystal structure of a Smad MH1 domain bound to DNA: insights on DNA binding in TGF-beta signaling. Cell 94, 585-594 (1998).

21. Burgstaller, G., Gregor, M., Winter, L. \& Wiche, G. Keeping the vimentin network under control: cell-matrix adhesion-associated plectin $1 \mathrm{f}$ affects cell shape and polarity of fibroblasts. Mol. Biol. Cell 21, 3362-3375 (2010).

22. Tsuruta, D. \& Jones, J. C. The vimentin cytoskeleton regulates focal contact size and adhesion of endothelial cells subjected to shear stress. J. Cell Sci. 116 4977-4984 (2003).

23. Wilson, D. C. et al. Follistatin-like protein 1 is a mesenchyme-derived inflammatory protein and may represent a biomarker for systemic-onset juvenile rheumatoid arthritis. Arthritis Rheum. 62, 2510-2516 (2010).

24. Clutter, S. D., Wilson, D. C., Marinov, A. D. \& Hirsch, R. Follistatin-like protein 1 promotes arthritis by up-regulating IFN-gamma. J. Immunol. 182, 234-239 (2009).

25. Oshima, Y. et al. Follistatin-like 1 is an Akt-regulated cardioprotective factor that is secreted by the heart. Circulation 117, 3099-3108 (2008).

26. Zarzynska, J. M. Two faces of TGF-beta1 in breast cancer. Mediat. Inflamm. 2014, 141747 (2014)
27. Sulzmaier, F. J., Jean, C. \& Schlaepfer, D. D. FAK in cancer: mechanistic findings and clinical applications. Nat. Rev. Cancer 14, 598-610 (2014).

28. Mitra, S. K. Hanson, D. A. \& Schlaepfer, D. D. Focal adhesion kinase: in command and control of cell motility. Nat. Rev. Mol. Cell Biol. 6, 56-68 (2005).

29. Shibue, T., Brooks, M. W., Inan, M. F., Reinhardt, F. \& Weinberg, R. A. The outgrowth of micrometastases is enabled by the formation of filopodium-like protrusions. Cancer Discov. 2, 706-721 (2012).

30. McLean, G. W. et al. The role of focal-adhesion kinase in cancer - a new therapeutic opportunity. Nat. Rev. Cancer 5, 505-515 (2005).

31. Frisch, S. M., Schaller, M. \& Cieply, B. Mechanisms that link the oncogenic epithelial-mesenchymal transition to suppression of anoikis. J. Cell Sci. $\mathbf{1 2 6}$ 21-29 (2013)

32. Hsia, D. A. et al. Differential regulation of cell motility and invasion by FAK. J. Cell Biol. 160, 753-767 (2003).

33. Havel, L. S., Kline, E. R., Salgueiro, A. M. \& Marcus, A. I. Vimentin regulates lung cancer cell adhesion through a VAV2-Rac1 pathway to control focal adhesion kinase activity. Oncogene 34, 1979-1990 (2015).

34. Kreis, S., Schonfeld, H. J., Melchior, C., Steiner, B. \& Kieffer, N. The intermediate filament protein vimentin binds specifically to a recombinant integrin alpha2/beta1 cytoplasmic tail complex and co-localizes with native alpha2/beta1 in endothelial cell focal adhesions. Exp. Cell Res. 305, 110-121 (2005).

35. Bhattacharya, R. et al. Recruitment of vimentin to the cell surface by beta3 integrin and plectin mediates adhesion strength. J. Cell Sci. 122, 1390-1400 (2009).

36. Mendez, M. G., Kojima, S. \& Goldman, R. D. Vimentin induces changes in cell shape, motility, and adhesion during the epithelial to mesenchymal transition. FASEB J. 24, 1838-1851 (2010). 